\title{
Accounting for Safety Barriers Degradation in the Risk Assessment of Oil and Gas Systems by Multistate Bayesian Networks
}

\author{
F. DIMAIO ${ }^{1}$, O. SCAPINELLO ${ }^{1}$, E. ZIO $^{1,2}$, C. $^{\text {CIARAPICA }}{ }^{3, *}$, S. $^{2}$ CINCOTTA $^{3}$, A. CRIVELLARI $^{3}$, \\ L. DECARLI ${ }^{3}$, L. LAROSA ${ }^{3}$
}

${ }^{1}$ Energy Department, Politecnico di Milano, Via La Masa 34, 20156 Milano (MI), Italy

${ }^{2}$ Mines ParisTech, PSL Research University, Sophia Antipolis, France

${ }^{3}$ Eni Natural Resources - HSEQ, via Emilia 1, 20097 San Donato Milanese (MI), Italy

\section{A R T I C L E I N F O}

\section{Keywords:}

Quantitative Risk Assessment

Living Risk Assessment

Bayesian Network

Safety Barrier

Key Performance Indicator

Probabilistic Safety Margins

\begin{abstract}
A B S T R A C T
In this paper, a multistate Bayesian Network (BN) is proposed to model and evaluate the functional performance of safety barriers in Oil and Gas plants. The nodes of the BN represent the safety barriers Health States (HSs) and the corresponding conditional Failure Probability (FP) values are assigned. HSs are assessed on the basis of specific Key Performance Indicators (KPIs) related to the barrier characteristics (i.e., technical, procedural or organizational, continuously monitored or event-based characterized). FP values are estimated from failure datasets (for technical barriers), evaluated by Human Reliability Analysis (HRA) (for operational and organizational barriers) and assigned by expert elicitation (for barriers lacking data or information). For illustration, the multistate BN model is developed for preventive barriers and applied to a case study related to the potential release of flammable material in the slug catcher of a representative O\&G Upstream plant which may lead to major accident scenarios (fire, explosion, toxic dispersion). The results from the case study demonstrate that the multistate BN model is able to account for the safety barriers HS and their associated functional performance.
\end{abstract}

\section{Introduction}

Processes in an Oil and Gas (O\&G) plant involve inherent hazards, which must be kept under control for safe operation. For this, safety by design is pursued with the implementation of preventive and mitigative safety barriers, for reducing the probability of accidents and mitigating their consequences, respectively (ISO 17776). Various approaches have been proposed to model the functional performance of safety barriers, including simplified risk indexes [11], Monte Carlo simulation [1] and Quantitative Risk Assessment (QRA) [31]. The latter is a commonly used framework, because of its comprehensiveness [18].

QRA is periodically performed throughout the life cycle of the plant to evaluate its safety status [26]. However, the techniques currently adopted (mainly Bow-Ties) [27] do not allow for a lean incorporation of the Knowledge, Information and Data (KID) [51] related to the health state of the components and systems, which become available during their life through inspections and measurements $[4,20,43]$. This leads to neglecting the degradation of components, equipment and safety barriers in the calculation of the risk measures. To equip QRA with the capability of incorporating newly available KID, various methodologies for this have been proposed in recent years, including Dynamic Risk Assessment (DRA) based on statistical failure data [46], Hidden Markov Gaussian Mixture Model (HM-GMM) for condition monitoring [44], DRA based on Hierarchical Bayesian Analysis [34] and time-dependent reliability analysis [17].

In this work, we advance further in this direction by proposing a multistate Bayesian Network (BN) approach [25,39] that embeds different types of KID in the assessment of the degradation of safety barriers and their functional performance, within a Living Risk Assessment framework. The use of BNs for risk assessment has been rapidly spreading $[2,3,8,28-30,35,45]$ and the novelty of the BN modelling here proposed is related to the comprehensive evaluation of the multistate variables, based on the new KID that becomes available in relation to the safety barriers Health States (HS), e.g., in the form of data from the monitoring system, information from field inspection and maintenance, knowledge analysis from reporting, etc. Different monitoring approaches (i.e., continuous monitoring, safety event reporting) and safety

\footnotetext{
* Corresponding author.

E-mail addresses: francesco.dimaio@polimi.it (F. DIMAIO), oscarscapinello@gmail.com (O. SCAPINELLO), enrico.zio@polimi.it (E. ZIO), costanza.

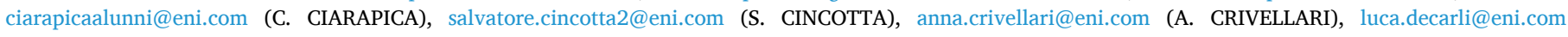
(L. DECARLI), laura.larosa@eni.com (L. LAROSA).
} 


\begin{tabular}{|c|c|c|c|}
\hline \multicolumn{2}{|c|}{ List of Acronyms } & $S_{-}^{i}$ & Family of states of the predecessors of node $i \in V$ \\
\hline ALARP & & $S_{+}^{l}$ & Family of states of the successors of node $i \in V$ \\
\hline $\begin{array}{l}\text { ALARP } \\
\text { PAPT }\end{array}$ & As Low As Reasonably Practicable & $P_{i}\left(s^{i}\right)$ & Probability of the event that node $i \in V$ is in state $s^{i} \in S^{i}$ \\
\hline $\begin{array}{l}\text { BART } \\
\text { BN }\end{array}$ & $\begin{array}{l}\text { BAseline Risk assessment Tool } \\
\text { Bayesian Network }\end{array}$ & $P_{i}\left(s^{i} \mid S_{-}^{i}\right)$ & Probability of the event that node $i \in V$ is in state $s^{i} \in S^{i}$ \\
\hline BT & Bow-Tie & & given the states of its predecessors \\
\hline CL & Checklist & $R$ & Reliability of a safety barrier evaluated by CL \\
\hline DI & Design Integrity & $T$ & Time horizon \\
\hline PDMP & Piecewise-Deterministic Markov Process & $\{\mathrm{H}, \mathrm{M}, \mathrm{L}\}$ & Set of HSs of a safety barrier node \\
\hline DRA & Dynamic Risk Assessment & $n_{i}$ & Number of incidents counted in a plant in $T=1$ year \\
\hline DyPASI & Dynamic Procedure for Atypical Scenario Identification & $X_{K P I}$ & Random variable describing the KPI \\
\hline ERS & Emergency Response System & $x_{K P I}$ & Observed value of $X_{K P I}$ in the range $[0,1]$ \\
\hline ESD & Emergency Shutdown & $X_{H S}$ & Random variable describing the HS of a safety barrier \\
\hline ET & Event Tree & $x_{H S}$ & State of $X_{H S}$, whose possible states are $\{\mathrm{H}, \mathrm{M}, \mathrm{L}\}$ \\
\hline FM & Fire Management & $a$ & Accident scenario \\
\hline FT & Fault Tree & $y$ & Safety parameter \\
\hline FP & Failure Probability & $\bar{y}(t)$ & Time series of parameter $y$ \\
\hline $\mathrm{H}$ & High (health state) & $U_{j}$ & Upper threshold \\
\hline HEP & Human Error Probability & $L_{j}$ & Lower threshold \\
\hline HRA & Human Reliability Analysis & $y_{\text {ref }}$ & Reference/Nominal value of parameter $y$ \\
\hline HS & Health State & $y_{\gamma}$ & $\gamma$-th percentile of $y$ distribution \\
\hline I\&D & Isolation and Depressurization & $\beta$ & Confidence of estimation \\
\hline KID & Knowledge, Information and Data & $\widehat{y}_{\gamma}$ & Estimated percentile \\
\hline KPI & Key Performance Indicator & $\Phi^{-1}(\cdot)$ & Inverse cumulative distribution function of the standard \\
\hline KPI\% & KPI percentile & & normal distribution \\
\hline $\mathrm{L}$ & Low (health state) & $Z_{L}$ & Low performance KPI threshold \\
\hline LOPC & Loss Of Primary Containment & $Z_{U}$ & High performance KPI threshold \\
\hline & Medium (health state) & $P\left(x_{H S} \mid x_{K P}\right.$ & PI) Probability of being in state $x_{\mathrm{HS}}$ given the assessed KPI \\
\hline MooN-G & M-out-of-N Good components & & value $x_{K P I}$ \\
\hline NHEP & Nominal Human Error Probability & $\left\{p_{H}, p_{M}, p_{L}\right.$ & ${ }_{4}$ \} Set of FPs corresponding to the HSs of a safety barrier \\
\hline OI & Operating Integrity & & node \\
\hline Os & Order Statistics & $p_{H S_{k}, k}$ & FP of the $k$-th barrier when found in $H S_{k} \in\{\mathrm{H}, \mathrm{M}, \mathrm{L}\}$ \\
\hline PCS & Process Control System & $P_{\text {LOPC }}(\cdot)$ & Leak probability in one year \\
\hline PPS & Pressure Protection System & $P_{i g n}$ & Ignition probability after a LOPC \\
\hline PSD & Process Shutdown & $P_{\text {imm }}$ & Immediate ignition probability \\
\hline PSF & Performance Shaping Factor & $P_{d e l}$ & Delayed ignition probability \\
\hline PSM & Probabilistic Safety Margin & $P_{e x}$ & Conditional explosion probability \\
\hline PSMS & Process Safety Management System & $P_{f f}$ & Conditional FF probability \\
\hline PSV & Pressure Safety Valve & $P_{\text {Cons }}(\cdot)$ & Probability of each consequence in one year \\
\hline QRA & Quantitative Risk Assessment & $\lambda_{\text {LOPC }}$ & Frequency of a LOPC accident (events/year) when no \\
\hline RI & Risk Indicator & & barrier is in place \\
\hline SCS & Spill Containment System & $\lambda_{L O P C, H S_{k}, k}$ & Frequency of a LOPC when the $k$-th barrier is in place and \\
\hline SD & Shutdown & & has a given $H S_{k} \in\{\mathrm{H}, \mathrm{M}, \mathrm{L}\}$ \\
\hline SHIPP & System Hazard Identification, Prediction And Prevention & B & Generic safety barrier \\
\hline TM & Task Management & $Q$ & Number of experts \\
\hline WH & Working Hours & $w_{q}$ & Weight assigned to the $q$-th expert, $q=1,2, \ldots, Q$ \\
\hline List of Sy & mbols & $\lambda_{L O P C, q}$ & Frequency of a LOPC accident (events/year) when no \\
\hline$V$ & Set of nodes of the Bayesian Network & & barrier is in place, as evaluated by the $q$-th expert \\
\hline$N$ & Number of nodes of the Bayesian Network & $\wedge_{L O P C, H S_{k}}$ & $q$ Frequency or a LUPC When the $k$-n Darrier is in piace \\
\hline$E$ & Set of arcs of the Bayesian Network & & and nas a given $H \triangle_{k} \in\{H, M, L\}$, as evaluated by the $q$-th \\
\hline$V_{-}^{i}$ & Set of predecessors of node $i \in V$ & & FP of the $k$-th barrier when is in place and has a given \\
\hline$V_{+}^{i}$ & Set of successors of node $i \in V$ & $P H S_{k}, k, q$ & $H S_{k} \in\{\mathrm{H}, \mathrm{M}, \mathrm{L}\}$, as evaluated by the $q$-th expert \\
\hline$S^{i}$ & Set of states of the event at node $i \in V$ & & \\
\hline
\end{tabular}

barrier types (i.e., technical, procedural, organizational) are considered and, for each of them, a tailored approach is developed to assess the barrier HS and the corresponding Failure Probability (FP) (i.e., the probability that the safety barrier in a specific HS does not perform its function). The probability $P(\cdot)$ of a barrier to be in a given HS (i.e., High $(\mathrm{H})$, Medium (M), Low (L)) is obtained with reference to specific Key Performance Indicators (KPIs) and based on the KID collected at the plant during a specific time [14]. The safety barrier FP can, then, be computed [16].

A case study consisting of a slug catcher of a representative Upstream O\&G onshore plant is presented to demonstrate the potential of the proposed multistate BN model. The results provided by the multistate BN model are compared with those obtained through the application of the BAseline Risk assessment Tool (BART) [40], which is the reference methodology for risk assessment developed and adopted by the global energy company Eni. 
The remainder of the paper is organized as follows: Section 2 presents the methodology background, introducing the BART procedure and the BN modelling features; Section 3 describes the procedure for the characterization of the safety barriers and Section 4 shows the application to the case study; finally, in Section 5 some conclusions are drawn.

\section{Methodological background}

\subsection{BAseline Risk assessment Tool Methodology}

BART combines a simplified QRA methodology with a Bow-Tie (BT) model to identify the potential hazards and evaluate the related risks that may arise from the processes and activities carried out in Upstream O\&G installations [10]. BT provides a graphical representation of accident scenarios, from causes to consequences (ISO 17776). It is comprised of a Fault Tree (FT) representing the possible events causing the accident, whose top event is the initiating event of an Event Tree (ET) delineating the scenarios that develop from it depending on the failure or success of the safety barriers $[19,5,12]$.

$\mathrm{BT}$ is used in BART to evaluate the actual risk posed by the potential accident scenarios whose baseline probability of occurrence is inferred from available documentation and databases (for example, the baseline yearly $P_{L O P C, 0}$ of a Loss of Primary Containment (LOPC) in a typical O\&G facility such as that considered in the case study can be assumed equal to $0.0241[22]$ ), accounting for the contribution to safety of the safety barriers placed in each key equipment unit for preventing and mitigating the accident escalation.

The analysis by BART involves:

1 Modelling the plant and its subdivision into key equipment units according to the process flow, the operating conditions and the equipment layout;

2 For each key equipment unit, specifying the consequences from potential accidents (e.g., Flash Fire, Jet Fire, Pool Fire, Explosion, Toxic Dispersion);

3 Evaluating the installation baseline risk category (Low, Medium, Medium-High, High) in terms of accident frequency and consequence severity, by the simplified QRA without considering any preventive and mitigative safety barrier in place;

4 Evaluating the actual risk category by BT methodology, considering the preventive and mitigative safety barriers in place (see Section 2.1);

5 Suggesting corrective actions for improving the reliability and functional performance of the safety barriers in place, or the addition of new safety barriers to reduce the overall exposure to risk.

The specific barrier contribution to safety is inferred from available documentation, plant personnel interviews and surveys. In particular, surveys are performed by filling checklists (CLs) and to qualitatively evaluate the reliability of each barrier. The CL survey is inevitably subjective and needs, thus, to be performed in a structured and controlled way. However, the BT framework has difficulty in accounting for the actual HS of the barriers in the evaluation of the actual risk. Finally, the static nature of the FTs and ETs in the BT does not allow to easily update the living risk assessment in view of the changes in time of the HS of the safety barriers in place.

\subsection{Bayesian Network methodology}

The method proposed in this work is an extension of BART, which involves the introduction of BNs to confer specific additional modelling capabilities. A BN is a probabilistic graphical based network that is represented as a directed acyclic graph [33] consisting of:

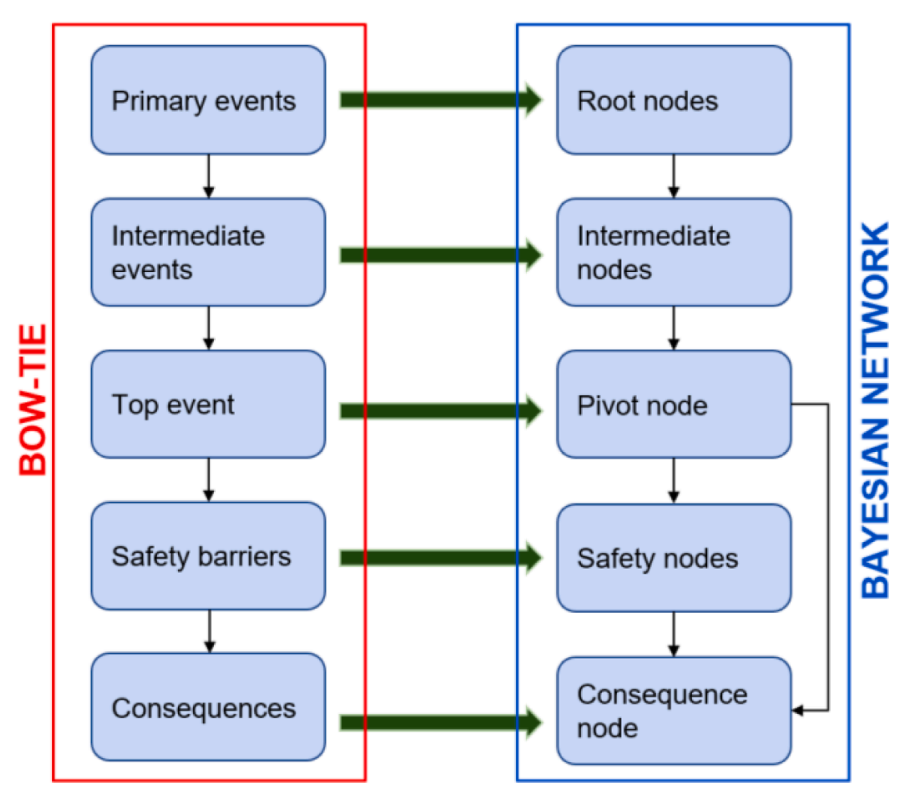

Figure 1. BT-BN mapping scheme adapted from [27]

- Nodes $V=\{1,2, \ldots, N\}$, which in our case represent events whose combinations can lead to an accident; so, each node $i$ represents a random event that encodes a set of discrete states $S^{i}=\left\{s_{1}^{i}, s_{2}^{i}, \ldots, s_{m}^{i}\right\}$ (multistate variables).

- Directed $\operatorname{arcs} E \subseteq\{(i, j) i, j \in V, i \neq j\}$, which indicate conditional dependencies among nodes. Specifically, the $\operatorname{arc}(j, i) \in E$, which connects node $j \in V$ to node $i \in V$, indicates that the event at node $i$ is conditionally dependent on the event at node $j$. A path is a sequence of nodes $\left(i_{1}, i_{2}, \ldots, i_{\eta}\right), \eta>1$, such that $\left(i_{j}, i_{j+1}\right) \in E$, $j<\eta$. The first node in the path, $i_{1}$, is called root node and the node $i_{\eta}$ is called leaf node of the path; the other nodes are called intermediate nodes. Since the BN is acyclic, there is no path $\left(i_{1}, i_{2}, \ldots, i_{\eta}\right)$ ,$\eta>1$ such that $\left(i_{j}, i_{j+1}\right) \in E, j<\eta$ and $i_{1}=i_{\eta}$. The immediate follower nodes of $i \in V$ form the set $V_{+}^{i}=\{j(i, j) \in E\}$ and their sets of states form the family $S_{+}^{i}=\left\{S^{j} j \in V_{+}^{i}\right\}$, whereas its immediate predecessor nodes are in the set $V_{-}^{i}=\{j(j, i) \in E\}$, whose set of states is the family $S_{-}^{i}=\left\{S^{j} j \in V_{-}^{i}\right\}$.

- Conditional Probability Tables (CPTs) assigned to the nodes, which quantify the conditional dependencies among nodes in terms of the conditional probability $P_{i}\left(S^{i} \mid S_{-}^{i}\right)$ that node $i$ will be in a particular state, given the states of its parent nodes.

The joint probability of a path of node states $\left(s^{1}, s^{2}, \ldots, s^{\eta}\right)$ in the BN leading to the consequence node $i_{\eta}$ is computed as:

$P\left(s^{1}, s^{2}, \cdots, s^{\eta}\right)=\prod_{j=1}^{\eta} P_{j}\left(s^{j} \mid s_{-}^{j}\right)$

\subsection{Mapping Bow-Ties into Bayesian Networks}

A BT may be converted into a BN following a specific mapping procedure, as described in [7,6] and [27]. The structure of the $\mathrm{BN}$ is developed from the FT of the BT in such a way that primary events, intermediate events and the top event of the FT are represented as the root nodes (initiating event node), intermediate nodes (safety nodes) and leaf node (consequence node) in the equivalent BN, respectively. Then, each safety barrier in the ET of the BT is represented by a safety node and the consequences of the accident scenarios of the ET are the states of the node. The BNs equivalent to the FT and ET of the BT are, then, connected to each other via the top event as a pivot node. Figure 1 


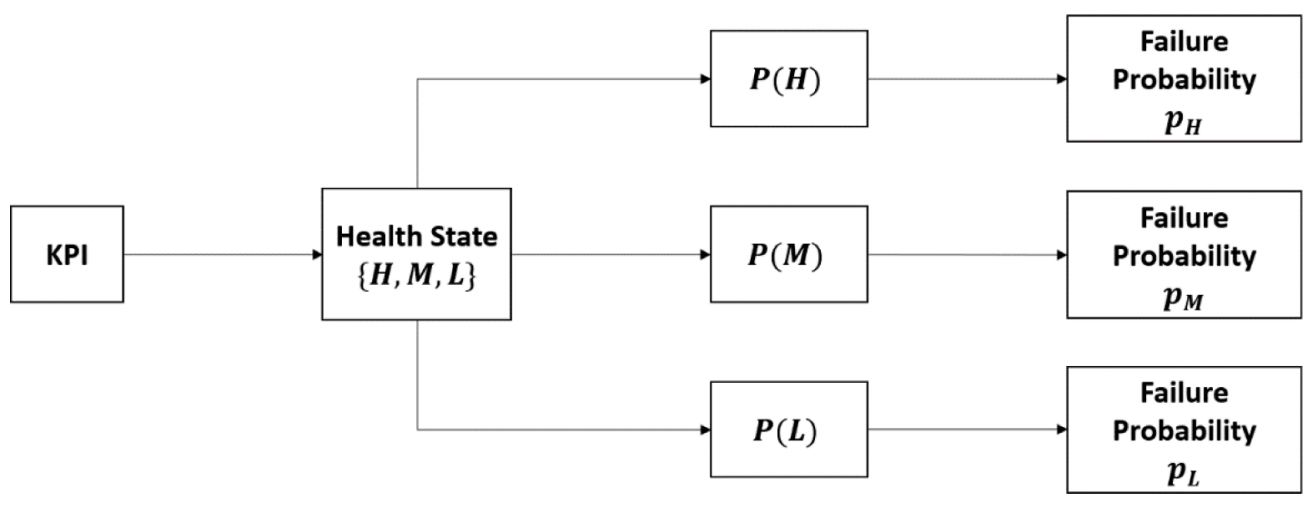

Figure 2. Barrier characterization concept scheme

Table 1

Anchor points for estimation of the safety barriers HS probability

\begin{tabular}{ll}
\hline & $\mathrm{P}\left(\mathrm{x}_{\mathrm{HS}} \mid x_{K P I}\right)$ \\
\hline 0 & $\{0.01,0.10,0.89\}$ \\
0.4 & $\{0.01,0.61,0.38\}$ \\
0.7 & $\{0.45,0.54,0.01\}$ \\
1 & $\{0.89,0.10,0.01\}$ \\
\hline
\end{tabular}

provides a scheme of the mapping procedure adopted.

\section{Safety barriers characterization}

The novelty of the BN model proposed in this work is that each safety barrier becomes a node in the BN and is described by the Health State (HS) and the Failure Probability (FP). The HS reflects the condition of the barrier with respect to its performance in fulfilling the function it is designed for. The HS is defined as a multistate variable, which can assume values as High (H), Medium (M) and Low (L), corresponding to nominal, acceptable and poor performance, respectively. Specifically, a safety barrier whose HS is H is considered to be in "as good as new" conditions, whereas if its HS is M or L it is considered to run in critical conditions (i.e., any further deterioration would prevent the barrier from working properly). A set of values $\{P(H), P(M), P(L)\}$ are defined as the probabilities that the HS is found in a specific state $\{\mathrm{H}, \mathrm{M}, \mathrm{L}\}$. Different approaches can be used to define $\{P(H), P(M), P(L)\}$ depending on the quality and quantity of the KID available, and to assign to each HS, based on specific Key Performance Indicators (KPIs), the probability that the barrier fails to perform its function when operating in a specific $\mathrm{HS}\{\mathrm{H}, \mathrm{M}, \mathrm{L}\}$, indicated as $p_{H}, p_{M}, p_{L}$, respectively.

Figure 2 shows a conceptualization of the safety barrier characterization approach, whose details will be provided in the following Sections.

\subsection{Health State probability estimation}

The HS reflects the condition of the barrier with respect to the performance of its safety function. It is defined as High (H), Medium (M) and Low (L), corresponding to nominal, acceptable and poor performance respectively. In the following paragraphs, a safety barrier whose HS is H is considered to be in "as good as new" conditions; a safety barrier whose HS is M is here modelled to be in critical conditions (i.e., each of its components is critical for it to work properly); a safety barrier whose HS is L is here modelled to be in a failed state.

The probability that the HS of a barrier is $\mathrm{H}, \mathrm{M}$ or $\mathrm{L}$ is quantified on the basis of specific KPIs, which are evaluated differently for the different safety barrier monitoring strategies (i.e., continuously monitoring or safety relevant event reporting), as explained in the following

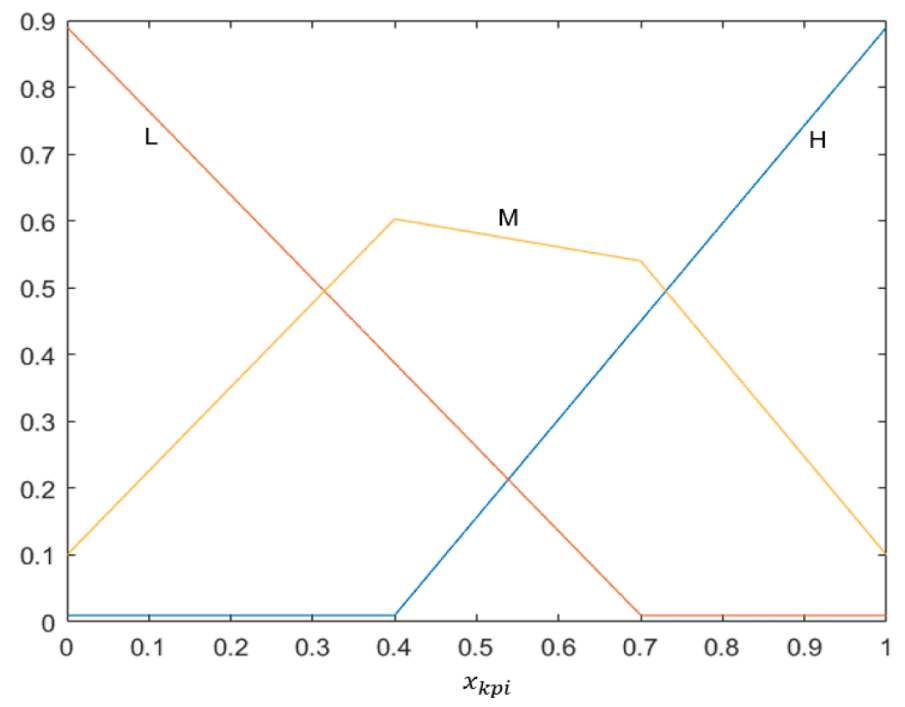

Figure 3. Probability distributions $\mathrm{P}\left(\mathrm{x}_{\mathrm{HS}} \mid \mathrm{x}_{\mathrm{KPI}}\right)$ for the HSs $\mathrm{H}, \mathrm{M}$ and $\mathrm{L}$

Sections. In line with [49], a KPI is described by continuous input variables $X_{K P I}$ with values $x_{K P I}$ in the range [0,1]. For a specific KPI value $x_{K P I}$ of $X_{K P I}$, there can be uncertainty with respect to the corresponding $\mathrm{HS}\{\mathrm{H}, \mathrm{M}, \mathrm{L}\}$ of the barrier. Then, the HS of a safety barrier is described by a discrete random variable $X_{H S}$, whose possible states are $\{\mathrm{H}, \mathrm{M}, \mathrm{L}\}$. To model the relationship between KPIs and HS $\{\mathrm{H}, \mathrm{M}, \mathrm{L}\}$, a probabilistic model is defined where expert-based prototypical conditions are used as anchor points. These points provide the analysts with examples of "nominal" assessment, in comparison of which acceptable and poor conditions can be assessed with respect to the deviations from the nominal anchors. For example, as shown in Table 1 and Figure 3, for the state $\mathrm{H}$ and anchor points) $x_{K P I}=0$ and $x_{K P I}=1$, an expert may judge $x_{K P I}=0$ to belong to HS $\{\mathrm{H}, \mathrm{M}, \quad \mathrm{L}\}$ with $P\left(x_{H S} \mid x_{K P I}\right)=$ $\{0.01,0.10,0.89\}$, respectively, and $x_{K P I}=1$ to belong to $\mathrm{HS}\{\mathrm{H}, \mathrm{M}, \mathrm{L}\}$ with $P\left(x_{H S} \mid x_{K P I}\right)=\{0.89,0.10,0.01\}$, respectively. The resulting distributions are shown in Figure 3. The probabilities $P\left(x_{H S} \mid x_{K P I}\right)$ are plotted for each HS according to the $x_{K P I}$ values: these distributions have been detained by linear interpolation between the anchor points. Depending on the kind of information available for the safety barrier of interest, several KPIs can be defined.

\subsubsection{KPIs for continuously monitored safety barriers}

Let $\bar{y}(t)=\left[y\left(t_{1}\right), y\left(t_{2}\right), \ldots, y\left(t_{n}\right)\right]$ be the data time series collected at the discrete times $t_{1}<t_{2}<\cdots<t_{n}$ from the continuous monitoring of the characteristic parameter of a specific safety barrier. Probabilistic Safety Margins (PSM) can be used to measure, in probabilistic terms, to 
which extent the safety parameter $y$ is below an acceptable upper threshold value $U$ (or above a lower threshold value $L$ ) during the monitoring interval $\left[t_{1}, t_{n}\right]$ [50]. This is done by calculating the estimate $\hat{y}_{\gamma}$ of the true $\gamma$-percentile $y_{\gamma}$ (usually the $95^{\text {th }}$ percentile) of the distribution of the parameter [13], with confidence $\beta$ (usually 95\%):

$\gamma=P\left\{y<y_{\gamma}\right\}$

$\beta=P\left\{y_{\gamma}<\widehat{y}_{\gamma}\right\}$

The estimate $\widehat{y}_{\gamma}$ thereby obtained is used to indicate to which extent the safety barrier has been in normal (i.e., $\mathrm{HS}=\{\mathrm{H}\}$ ) or degraded (i.e., $\mathrm{HS}=$ $\{\mathrm{M}, \mathrm{L}\})$ conditions during the monitoring interval $\left[t_{1}, t_{n}\right]$. The calculation of $\widehat{y}_{\gamma}$ can be done by empirical percentile estimation methods (in case of sufficient monitoring data) or by Order Statistics (OS) [37], which provides the minimum number $S$ of samples needed to obtain the estimate $\widehat{y}_{\gamma}$ with the desired confidence $\beta$. Without loss of generality, considering the safety parameter $y$ to be limited from above by the upper threshold $U$ (the extension to the lower threshold $L$ being straightforward), the $m$-th member $y_{m}$ of the $S$ samples sorted by descending value has a certain probability $\beta$ of exceeding the unknown true $\gamma$-th percentile $y_{\gamma}$. Then, one can get the level of confidence $\beta$ that the actual value of $y_{\gamma}$ is less than the value obtained for $y_{m}$ and if $y_{m}$ meets the criterion of being less than the threshold $U$, then the unknown $y_{\gamma}$ will do so, too.

For an upper threshold $U$, the PSM can be calculated as [13]:

$P S M=\left\{\begin{array}{c}\frac{U-\widehat{y}_{\gamma}}{U-y_{\text {ref }}} \quad \text { if } \hat{y}_{\gamma} \leq U \\ 0 \text { if } \hat{y}_{\gamma}>U \\ 1 \text { if } \hat{y}_{\gamma}<y_{\text {ref }}\end{array}\right.$

and for the lower threshold $L$, as:

$P S M=\left\{\begin{array}{c}\frac{\widehat{y}_{\gamma}-L}{y_{\text {ref }}-L} \quad \text { if } \widehat{y}_{\gamma} \geq L \\ 0 \text { if } \hat{y}_{\gamma}<L \\ 1 \text { if } \hat{y}_{\gamma}>y_{\text {ref }}\end{array}\right.$

where $y_{\text {ref }}$ is a reference value for $y$, which is typically the nominal value of the parameter.

The PSM thus evaluated is, then, taken as $x_{K P I}$ for the evaluation of the probability of continuously monitored technical barriers to be in given $H S \in\{H, M, L\}[15]$.

\subsubsection{KPIs for event-based safety barriers}

Let us assume that event data from various sources have been accumulated for a certain period of time, which can be used for calculating event-based KPIs (for example, number of accidents, alarm counts, number of Emergency Shutdowns (ESDs) or Process Shutdowns (PSDs)). For each KPI, a specific probabilistic distribution is defined: KPI values indicating low performance are made to correspond to the low percentiles of said distribution, and KPI values indicating high performance to correspond to high percentiles of the distribution. For instance, let us assume that a particular KPI follows a normal distribution $N\left(\mu, \sigma^{2}\right)$ [41] and its performance is considered poor for KPI values below a specific threshold, $Z_{L}$, and good for KPI values above a different threshold, $Z_{U}$. The parameters $\mu$ and $\sigma$ of the normal distribution can be evaluated from the following system of equations:
$\left\{\begin{array}{l}\frac{Z_{L}-\mu}{\sigma}=\Phi^{-1}(0.4) \\ \frac{Z_{U}-\mu}{\sigma}=\Phi^{-1}(0.7)\end{array}\right.$

which leads to:

$\left\{\begin{array}{c}\mu=\frac{\Phi^{-1}(0.7) Z_{L}-\Phi^{-1}(0.4) Z_{U}}{\Phi^{-1}(0.7)-\Phi^{-1}(0.4)} \\ \sigma=\frac{Z_{U}-Z_{L}}{\Phi^{-1}(0.7)-\Phi^{-1}(0.4)}\end{array}\right.$

where $\Phi^{-1}(\cdot)$ is the inverse cumulative distribution function of the standard normal distribution and the $40^{\text {th }}$ and $70^{\text {th }}$ percentiles are chosen from the anchor points reported in Table 1.

Once the distribution is completely defined, it is possible to associate each new KPI score to a percentile (KPI\%). The KPI\% is, then, taken as $x_{K P I}$ for the evaluation of the probability of the safety barrier HS to be one of $\{\mathrm{H}, \mathrm{M}, \mathrm{L}\}$.

\subsection{Failure Probability Evaluation}

The probability FP that the barrier fails to perform its function in a considered period of time when its $\mathrm{HS}$ is $\mathrm{H}, \mathrm{M}$ or $\mathrm{L}$, is indicated as $p_{H}$, $p_{M}, p_{L}$, respectively. Depending on the type of barrier (technical, procedural or organizational) a different procedure has been devised for estimating these probabilities:

- Technical: estimation of failure rates from existing databases, such as [38] (see Section 4.2.1);

- Procedural: estimation of failure rates by Human Reliability Analysis (HRA) (see Section 4.2.2);

- Organizational: estimation of failure rates by Organizational and Human Reliability Analysis (OHRA) (see Section 4.2.2).

When data relative to some specific barriers are missing, the FP estimation is done by expert elicitation, as proposed in $[16,42]$.

\subsubsection{FP assessment for technical barriers}

The FP estimation for a technical barrier for which failure rates can be retrieved from existing databases proceeds by first performing a functional analysis of the safety barrier by a top-down approach, aimed at identifying the components of the barrier and understanding the functional logic. For each component of the barrier, a failure rate $\lambda$ encompassing all causes of failure is retrieved from existing databases, for example [38]. Since barrier components are periodically inspected at regular intervals T, the Probability of Failure on Demand (PFD) for a single component can be used as follows (Zio, 2007):

$\mathrm{PFD}=\frac{1}{2} \lambda \mathrm{T}$

Then, the FP of the whole barrier is calculated, depending on the logical architecture which the components are organized in. If the barrier logical architecture can be modelled as a MooN-G (M-out-of-N Good components) configuration, in practice, the FPs $\left\{\mathrm{p}_{\mathrm{H}}, \mathrm{p}_{\mathrm{M}}, \mathrm{p}_{\mathrm{L}}\right\}$ corresponding to the $\mathrm{HS}\{\mathrm{H}, \mathrm{M}, \mathrm{L}\}$ are evaluated assuming that the barrier is as good as new (for $\mathrm{H}$ ), some components of the barrier are in degraded conditions (for $\mathrm{M}$ ), the barrier is in such low functional conditions that it cannot perform its function (for $\mathrm{L}$ ).

For example, for a safety barrier designed in the 2003-G architecture, which implies that 2 out of 3 elements must work (G) for the safety barrier to fulfill its function, it is $p_{H}=\lambda^{2} T^{2}$, when its KPI is indicating that HS is $\mathrm{H}, p_{M}=\lambda T$, if the KPI indicates that one of the three components is failed resulting in a 2002 configuration, and, finally, $p_{L}=1$ when $H S=L$ (failed barrier state). For barriers, whose logical archi- 


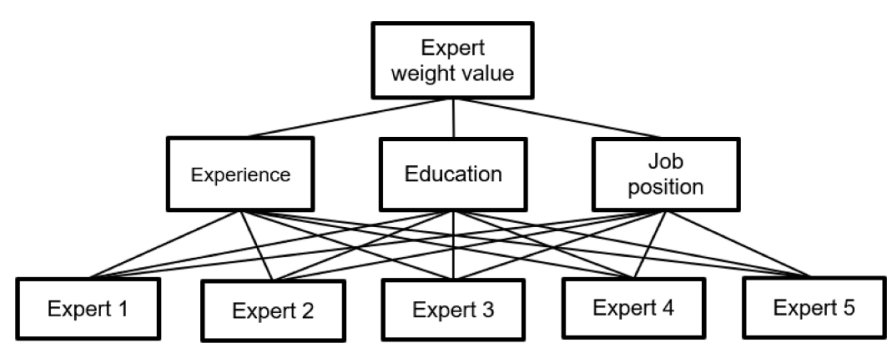

Figure 4. AHP model for the expert weight elicitation

tecture cannot be modelled as MooN-G systems, other logic methods can be used, e.g. FT analysis [21]. FT analysis entails the definition of an undesired event (e.g., failure of the barrier) as top event, whose causes are decomposed down to the basic events at the level of the system components. The probabilities of the basic events are estimated from databases [38] and following the Boolean logic of the FT, the probability of occurrence of the top event (i.e., the FPs of the barrier) is estimated.

\subsubsection{Failure Probability assessment for organizational and procedural barriers}

Procedural and organizational barriers failures are mainly due to human errors, whose probabilities can be obtained through HRA [36]. Several HRA methods have been developed and used in practice (e.g. THERP [32], CREAM [23], SPAR-H [19]), all aimed at the quantification of the Human Error Probability (HEP), which is usually considered as dependent on Performance Shaping Factors (PSFs) that define the stress conditions under which personnel tasks are to be performed.

In this work, the SPAR-H method for HEP calculation is adopted as follows:

$$
H E P=\frac{N H E P \times \prod_{1}^{n} P S F_{u}}{N H E P \times\left(\prod_{1}^{n} P S F_{u}-1\right)+1}
$$

where NHEP is the Nominal HEP value, which can be found in (IOGP 434-5) and $P S F_{u}, u=1,2, \ldots, n$, are the PSF values accounting for, for example, workload, stress, time available, training and experience. Depending on the effect of the PSFs, $p_{H}, p_{M}$ and $p_{L}$ can be evaluated as follows:

- If the PSFs degrade the HEP, then HEP $>N H E P$ and $p_{H}=N H E P$ when the barrier KPI indicates that HS is equal to $\mathrm{H}, p_{M}=H E P$ if the KPI indicates that $\mathrm{HS}$ is $\mathrm{M}$, and finally $p_{L}=1$ when the KPI indicates that the HS is equal to L (failed barrier);

- If incidentally the PSFs happen to improve the barrier effectiveness, then $H E P \leq N H E P$ and $p_{H}=H E P$ when the barrier KPI indicates that
HS is equal to $\mathrm{H}, p_{M}=N H E P$ if the KPI indicates that HS is M, and finally $p_{L}=1$ when the KPI indicates that the HS is equal to L (failed barrier).

\subsubsection{Failure Probability assessment for barriers lacking data}

When data are not available, an expert elicitation procedure is resorted to quantify $p_{H}, p_{M}$ and $p_{L}$ [42]. In practice, $Q$ experts are questioned in order to collect their educated assignment on the FP values $p_{H S, q}$ of a safety barrier for the different $\mathrm{HS}\{\mathrm{H}, \mathrm{M}, \mathrm{L}\}$ and, then, the expert values are weight-aggregated as:

$$
p_{H S}=\sum_{q=1}^{Q} p_{H S, q} w_{q}
$$

where $w_{q}$ is the weight assigned to the $q$-th expert, $q=1,2, \ldots, Q$, which accounts for various factors influencing his/her credibility (e.g., education level, professional position and years of experience) ([47]; Zarei et al., 2017). The expert relative weights can be obtained by applying the Analytic Hierarchy Process (AHP) (Figure 4), with pairwise comparisons of the trustworthiness of the experts (Yazdi et al., 2017).

Comparison data are collected into comparison matrices. Each element $c_{i j}$ of a comparison matrix $C$ should satisfy the constraint $c_{i j} \cdot c_{j i}=$ 1 .The weights relative to the importance for each entry (expert or criterion) can be determined by solving an eigenvector problem. More precisely, it can be shown that given the matrix of pairwise comparisons for the element of interest, the principal eigenvector provides the vector of weights, when normalized, and the maximum eigenvalue is a measure of consistency of the comparisons entered in the matrix [48]. For complete consistency, the maximum eigenvalue, $\lambda_{\max }$, should be equal to the order of the matrix, $m$. The level of consistency of a given pairwise comparison matrix can be measured by a parameter called Consistency Ratio (CR), defined as the ratio between the Consistency Index, $C I=\left(\lambda_{\max }-m\right) /(m-1)$, and the random index (RI), which is the statistically averaged $C I$ of randomly generated matrices of order $m$ with entries artificially forced to be consistent. A consistency ratio of 0.10 or less is considered acceptable in many practical applications (Saaty, 1980).

\section{Case study}

An application of the proposed methodology is presented in this Section, with respect to the slug catcher unit of a representative Upstream O\&G onshore plant. The slug catcher receives the incoming multiphase flow from the offshore platforms and performs a preliminary

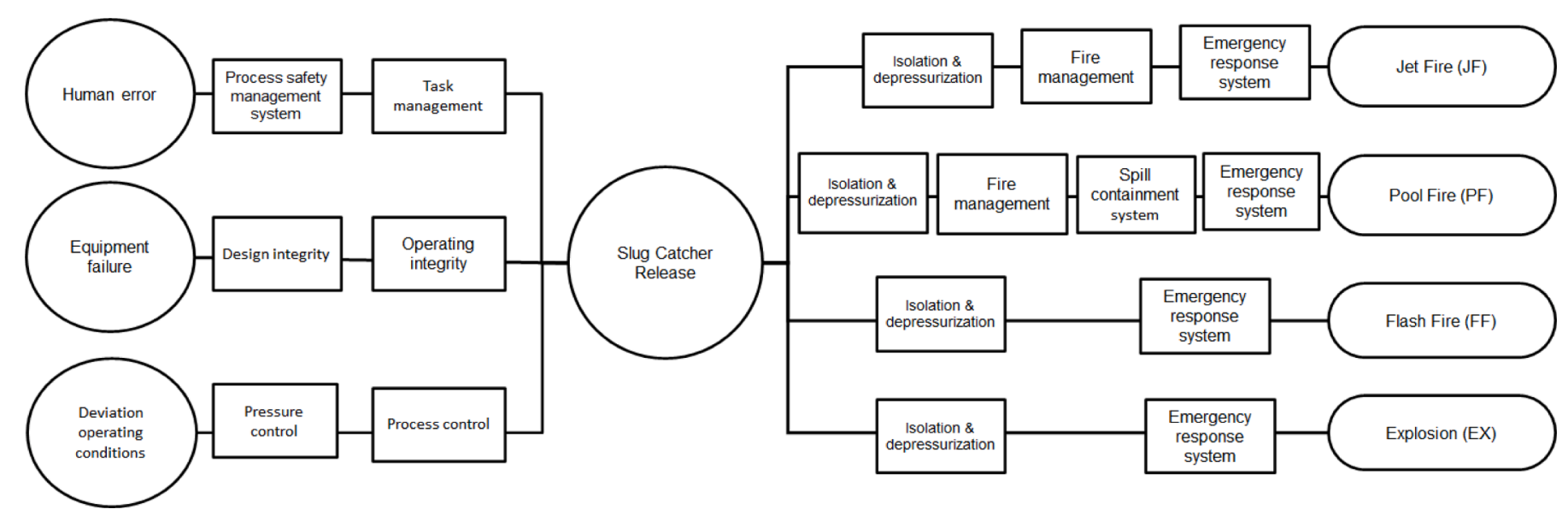

Figure 5. Slug catcher BT 


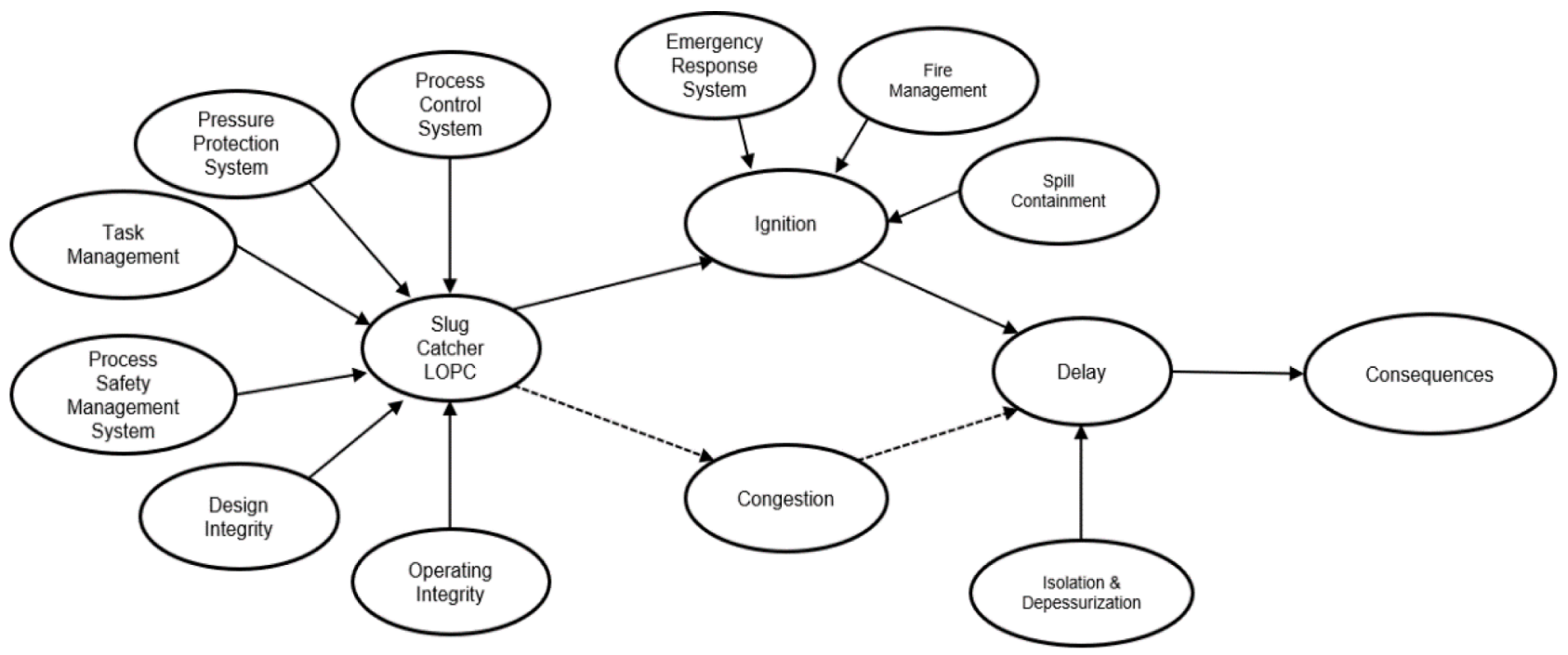

Figure 6. BN of the slug catcher LOPC

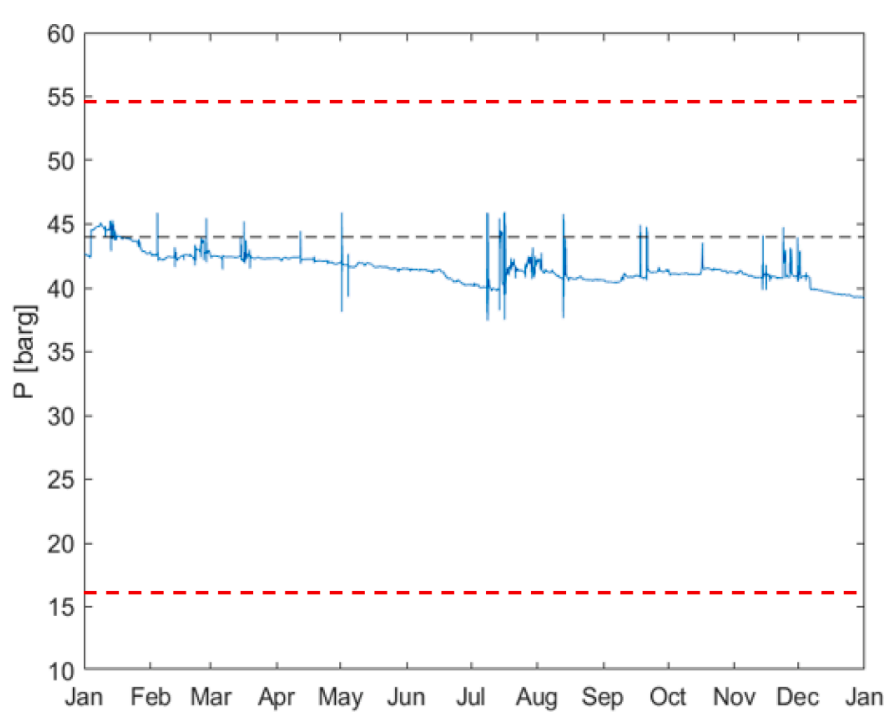

Figure 7. Example of pressure trend of the slug catcher in one-year operation. Dotted lines show nominal pressure (black), upper and lower thresholds (red)

gas liquid separation, thus representing the first process bottleneck of the onshore facility. The results of the application are benchmarked with those obtained by BART, whose top event is defined as the LOPC of the slug catcher, which can lead to different consequences, namely Flash Fire, Jet Fire, Pool Fire and Explosion. BART uses a BT for the LOPC of the slug catcher (shown in Figure 5): several hazards (e.g., human error, equipment failure and deviation from operating conditions) are modelled to lead to the top event "Slug Catcher LOPC" (circle in the middle); some preventive safety barriers (e.g., Process Safety Management System (PSMS), Task Management (TM), Design Integrity (DI), Operating Integrity (OI), Process Control System (PCS) and Pressure Protection System (PPS)) are considered to reduce the LOPC probability, and some mitigative barriers (e.g., Isolation \& Depressurization (I\&D), Fire Management (FM), Emergency Response System (ERS) and Spill Containment System (SCS)) are considered to mitigate the effects of the accident consequences (final leaves).

Following the BT-BN mapping procedure of Section 3.1, the BN shown in Figure 6 is built. The pivot node "Slug catcher LOPC", fed by the preventive safety barriers nodes, leads to the intermediate nodes describing the ignition escalation, fed by the mitigative barriers, and ultimately to the consequence node. In what follows, the preventive safety barriers are characterized according to the procedures defined in Section 4.

\subsection{Preventive safety barriers Health State characterization}

\subsubsection{Continuously monitored preventive safety barriers}

The PCS of the slug catcher is a monitored safety barrier that keeps the system pressure within the operational range and as close as possible to the nominal pressure value. As seen in Section 3.1.1, PSMs can be defined as a KPI for HS evaluation. Let us consider the time series $\bar{y}(t)$ plotted in Figure 7 , consisting of $n=1770$ data collected over a monitoring time of one year: the monitored slug catcher works at a nominal pressure set at 44 barg. Thresholds are set with respect to the design pressure of the slug catcher: the upper threshold $(U)$ at 54 barg; the lower threshold $(L)$ at 16 barg.

Resorting to OS, for $\gamma=95 \%, \beta=95 \%$ and $m=1$ (see Section 4.1.1), the PSM for the lower $\left(\mathrm{PSM}_{\mathrm{L}}\right)$ and the upper $\left(\mathrm{PSM}_{\mathrm{U}}\right)$ thresholds are $\mathrm{PSM}_{\mathrm{L}}=0.771$ and $\mathrm{PSM}_{\mathrm{U}}=0.840$. To be conservative, the lowest PSM value is used to evaluate the HS probabilities, leading to $P_{P C S}(H)=0.55$, $P_{P C S}(M)=0.44$ and $P_{P C S}(L)=0.01$. These values are introduced into the $\mathrm{BN}$ in order to fill the CPT of the PCS.

\subsubsection{Event-based preventive safety barriers}

For the TM of the slug catcher, assuming that its performance follows a normal distribution, a KPI has been defined as obeying to a normal distribution $\mathrm{N}(20,19)$; the KPI evaluated during operations is KPI = 12.58 based on reporting and the corresponding percentile of the normal distribution is KPI $=0.65$. Then, as described in Section 4.1, the probability that the barrier is found in a particular $\mathrm{HS}\{\mathrm{H}, \mathrm{M}, \mathrm{L}\}$, given the KPI evaluation is calculated with the probabilistic relationship of Figure 2, resulting in $P_{T M}(H)=0.62, P_{T M}(M)=0.37$ and $P_{T M}(L)=0.01$.

Likewise, several KPIs and their corresponding distributions have been defined for the remaining preventive barriers (namely PPS, PSMS, OI and DI) and their HS probability distributions are shown in the CPTs in Figure 9.

\subsection{Preventive safety barriers Failure Probability characterization}

\subsubsection{Technical preventive safety barriers}

Two technical barriers are implemented for the slug catcher pressure safety, namely the PPS and the PCS. The FP $\left\{p_{H}, p_{M}, p_{L}\right\}$ calculation of the PPS technical barrier, whose components are assumed to have constant failure rates with values estimated from data of available da- 


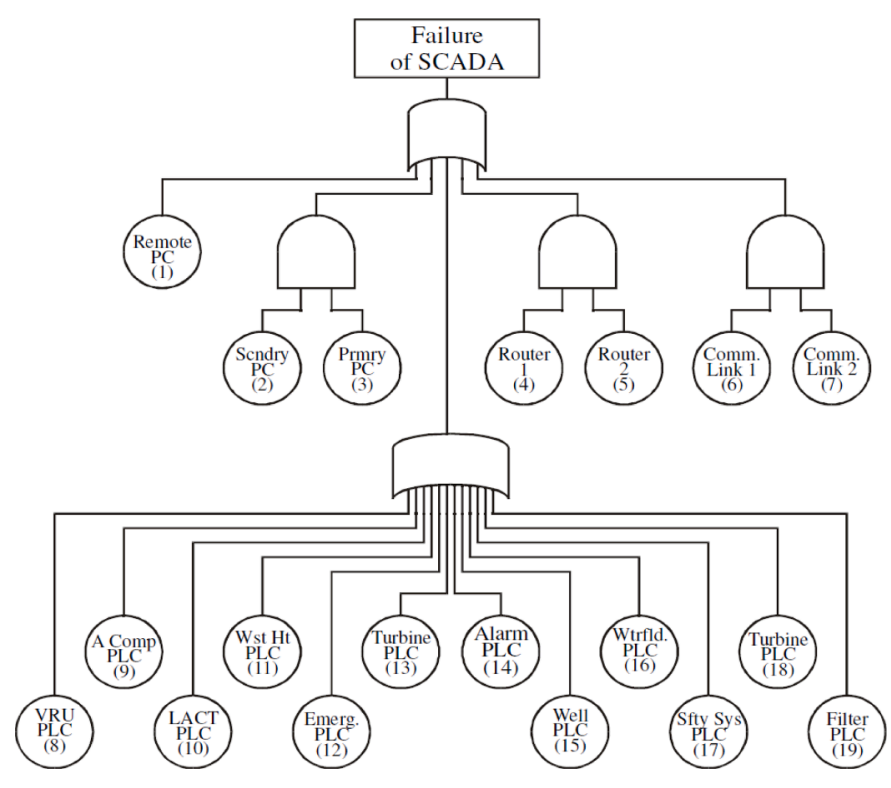

Figure 8. Fault Tree of the typical SCADA system [9]

Table 2

SCADA components failure data [9]

\begin{tabular}{ll}
\hline Component & PFD $_{\text {AvG }}$ \\
\hline PC & 0.0005 \\
Router & 0.0000004 \\
Comm. Link & 0.01 \\
PLC & 0.00015 \\
\hline
\end{tabular}

Table 3

Expert scores and weights from the application of the expert elicitation approach

\begin{tabular}{lllll}
\hline Expert & Job & Experience & Education & $\mathrm{w}_{\mathrm{q}}$ \\
\hline Expert 1 & 3 & 3 & 4 & 0.20 \\
Expert 2 & 2 & 1 & 4 & 0.06 \\
Expert 3 & 2 & 1 & 4 & 0.06 \\
Expert 4 & 2 & 2 & 4 & 0.10 \\
Expert 5 & 2 & 1 & 5 & 0.08 \\
Expert 6 & 2 & 2 & 4 & 0.10 \\
Expert 7 & 2 & 1 & 4 & 0.06 \\
Expert 8 & 2 & 1 & 4 & 0.06 \\
Expert 9 & 2 & 2 & 4 & 0.10 \\
Expert 10 & 2 & 1 & 5 & 0.08 \\
Expert 11 & 2 & 2 & 4 & 0.10 \\
\hline
\end{tabular}

tabases, such as [38], proceeds as described in Section 4.2.1 by firstly modelling the logical configuration of the barrier. The PPS of the slug catcher is considered to be composed of two Pressure Safety Valves (PSVs) $\left(\lambda_{P S V}=3.8 \cdot 10^{-5} y^{-1}\right.$, from [38]) in 1002 configuration, meaning that at least one PSV must work correctly for the overall system success. When both PSVs are healthy (HS equal to H), the system preserves its 1oo2-G architecture and $p_{H, P P S}=\frac{\lambda_{P S V}^{2} T^{2}}{3}=0.0375$; in case one of the PSVs is not working (HS equal to $\mathrm{M}$ ) for any reason (e.g., due to degradation, interlock position or failure), the PPS is turned into a 10o1-G system, whose $p_{M, P P S}=\frac{\lambda T}{2}=0.1504$; when both PSVs are failed (HS equal to L) $p_{L, P P S}=1$.

As for the FP $\left\{p_{H}, p_{M}, p_{L}\right\}$ calculation of the PCS, the FT of a typical Supervisory Control and Data Acquisition (SCADA) system operating the PCS is considered (Figure 8).

The $\mathrm{PFD}_{\mathrm{AVG}}$ values for the components failure are taken from [9] and listed in Table 2.

The evaluated FPs are, therefore, $p_{H, P C S}=0.0024$, when HS is equal
Table 4

Expert answers from the application of the expert elicitation approach

\begin{tabular}{llll}
\hline Expert & $\lambda_{\mathrm{LOPC}, q}$ & $\lambda_{\mathrm{LOPC}, \mathrm{H}, \mathrm{OI}, q}$ & $\lambda_{\mathrm{LOPC}, \mathrm{M}, \mathrm{OI}, \mathrm{q}}$ \\
\hline Expert 1 & 5 & 1 & 4 \\
Expert 2 & 100 & 30 & 70 \\
Expert 3 & 100 & 80 & 90 \\
Expert 4 & 100 & 80 & 90 \\
Expert 5 & 0.14 & 0.12 & 0.13 \\
Expert 6 & 10000 & 0.0001 & 0.01 \\
Expert 7 & 20 & 9 & 11 \\
Expert 8 & 100 & 25 & 75 \\
Expert 9 & 50000 & 5000 & 0.01 \\
Expert 10 & 10000 & 0.0001 & 0.001 \\
Expert 11 & 100000 & 0.0001 & 0.01 \\
\hline
\end{tabular}

to $\mathrm{H}, p_{M, P C S}=0.0182$, when $\mathrm{HS}$ is equal to $\mathrm{M}$ (i.e., redundancies have been ignored) and $p_{L, P C S}=1$ for $\mathrm{HS}$ equal to $\mathrm{L}$.

\subsubsection{Preventive safety barriers with missing data}

No data are available for the non-technical safety barriers of the case study. Therefore, the corresponding FPs, $p_{H}, p_{M}$ and $p_{L}$ are evaluated resorting to an expert elicitation procedure as described in Section 4.2.3. A group of $Q=11$ experts has been surveyed: their personal factors (education level, professional position and years of experience) are scored and used to evaluate their $w_{q}$ (see Table 3 ).

According to the aggregation procedure described in Section 4.2.3, the values of $p_{H, O I, q}$ and $p_{M, O I, q}$ are computed for each expert $q=1,2$, $\ldots, Q$ from the answers listed in Table 4. Applying the $w_{q}$ shown in Table 3 and Eq. (10), $p_{H, O I}=0.311$ and $p_{M, O I}=0.492$ are calculated, whereas it is assumed that $p_{L, O I}=1$.

In a similar fashion, it is possible to elicit the FP values for the remaining non-technical safety barriers (namely TM, PSMS and DI), for which no data are available, in order to fill their corresponding CPTs. The results of the elicitation process for these barriers are shown in Figure 9, where the BN is completed with the CPTs for each preventive safety barrier and their effects on the system are considered in the consequence node risk assessment.

\subsection{Risk assessment}

Once the barriers have been fully characterized with respect to the values of HS, on the basis of the KID collected during a specific monitoring period (e.g., one year), the probability of the consequences, $P_{\text {Cons }}(\cdot)$, can be evaluated following the $\mathrm{BN}$ rules (in Appendix A, the procedure is described in details). The results provided by the multistate BN-based approach with the CPTs of Table 5 are shown in Figure 9. They are compared with the results of the BT-based approach of BART, reported in Table 6. Both approaches provide coherent risk results (i.e., low risk).

To show the applicability of the proposed multistate BN model for Living QRA, let us assume that, after one year of operation of the slug catcher, the KID with respect to each safety barrier are collected, thus allowing to update the KPIs (resulting in the values listed in Table 7). The HSs of each safety barrier are correspondingly updated and fed to the $\mathrm{BN}$, which generates the updated risk assessment (Figure 10). Without loss of generality, for the KPIs here considered, DI and PPS have shown better performance with respect to the previous monitoring period of one year, and OI, PCS,TM and PSMS, have shown worse performance with respect to the previous monitoring period: then, the final probabilities of the consequences Flash Fire, Explosion, Pool Fire, Jet Fire have increased from $9 \cdot 10^{-7}, 1 \cdot 10^{-7}, 8 \cdot 10^{-7}, 2 \cdot 10^{-7}$ to $4 \cdot 10^{-6}, 4$. $10^{-6}, 3 \cdot 10^{-6}, 7 \cdot 10^{-7}$, respectively. Few safety barriers have improved their KPIs whereas most have worsened their performance, leading to a larger consequence probability than at the previous inspection time: this is a key information from the Living QRA conditioned on the KID 


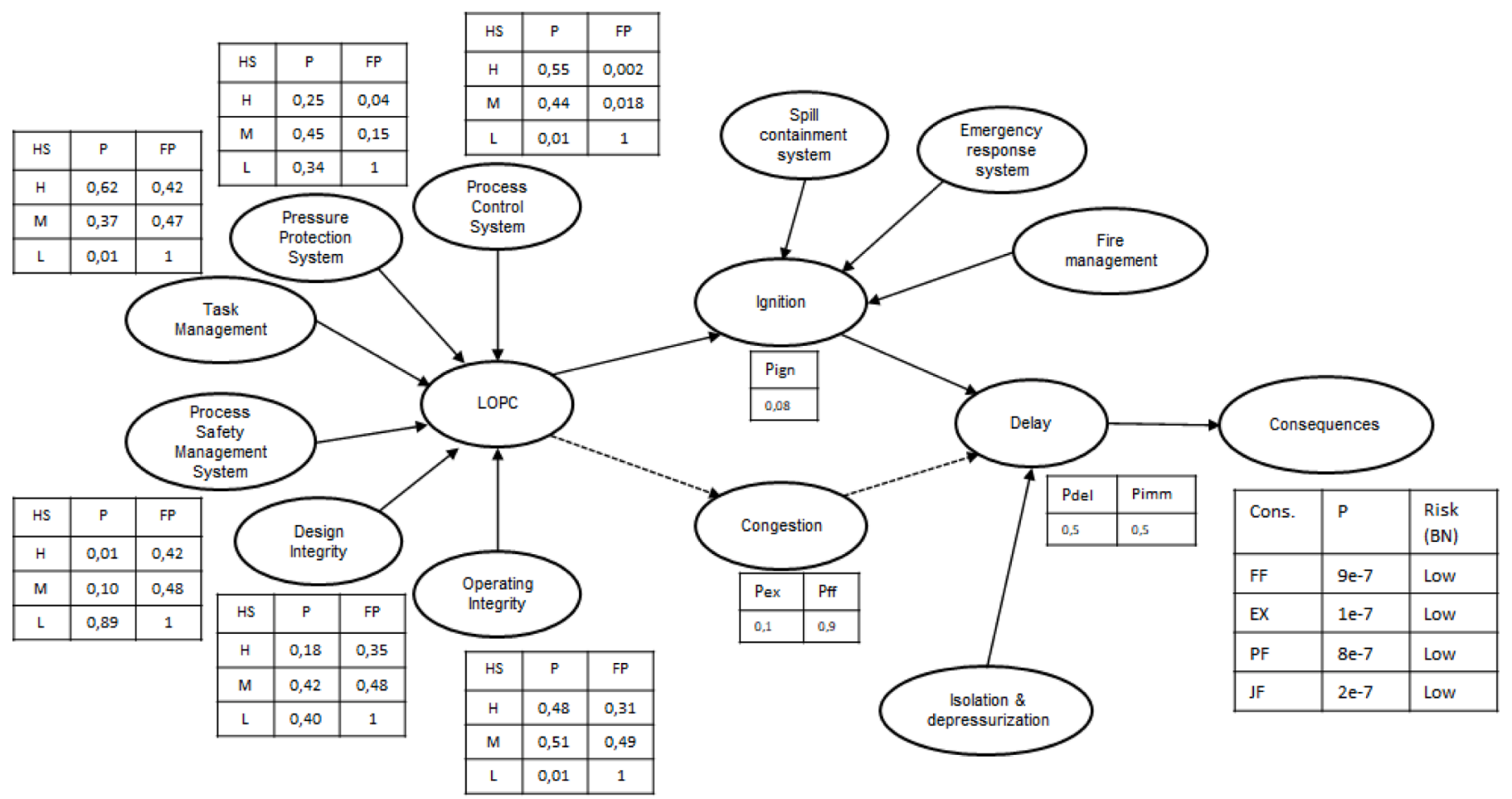

Figure 9. BN complete with safety barriers CPTs

Table 5

Preventive safety barriers $\mathrm{P}(\mathrm{HS})$ and FP triplets

\begin{tabular}{lll}
\hline Barrier & $\mathrm{P}(\mathrm{HS})$ & $\mathrm{FP}$ \\
\hline OI & $\{0.48,0.51,0.01\}$ & $\{0.31,0.49,1\}$ \\
PSMS & $\{0.01,0.10,0.89\}$ & $\{0.42,0.48,1\}$ \\
PCS & $\{0.54,0.45,0.01\}$ & $\{0.002,0.018,1\}$ \\
PPS & $\{0.25,0.41,0.34\}$ & $\{0.04,0.15,1\}$ \\
TM & $\{0.62,0.37,0.01\}$ & $\{0.01,0.10,1\}$ \\
DI & $\{0.18,0.42,0.40\}$ & $\{0.35,0.45,1\}$ \\
\hline
\end{tabular}

Table 6

Risk of each consequence evaluated by both BT-based and BN-based approaches

\begin{tabular}{lll}
\hline Consequence & Risk (BT) & Risk (BN) \\
\hline Flash Fire & LOW & LOW \\
Explosion & LOW & LOW \\
Jet Fire & LOW & LOW \\
Pool Fire & LOW & LOW \\
\hline
\end{tabular}

Table 7

KPIs (both previous and updated) of the preventive safety barriers

\begin{tabular}{lll}
\hline Safety barrier & KPI (previous) & KPI (updated) \\
DI & 0.588 & 0.608 \\
OI & 0.704 & 0.646 \\
PCS & 0.771 & 0.518 \\
PPS & 0.356 & 0.693 \\
PSMS & 0.002 & 0.001 \\
TM & 0.822 & 0.775 \\
\hline
\end{tabular}

collected on the system, and follow-up decisions can be taken to best manage the updated risk conditions.

\section{Conclusions}

In this work, a BN-based modelling approach for living QRA is proposed, which allows the dynamic assessment of safety barriers as their functional performance changes in time. The added value of the approach consists in the accommodation, within the BN framework, of multistate variables for describing safety barriers degradation, which is described in terms of HS definition and the corresponding FP. The analysis of the barriers HS is customized depending on their condition monitoring: continuously monitored barriers are described by PSMs, which allow accounting for uncertainties through confidence intervals and continuously updating KPIs for the HS evaluation; non-continuously monitored barriers are described by integral KPIs, each one related to a specific distribution of the number of events. FPs are evaluated depending on the type of barrier (technical, procedural or organizational), giving the necessary versatility and depth to the BN-based approach for realistic barrier modelling.

This work has shown the capability of BNs to handle multistate variables for modelling barrier behaviour under different HSs at different times, and calculating the corresponding FPs. HSs and FPs have been set relying on KPIs and procedures specifically tailored for each safety barrier, and allowing for a deep and realistic analysis of the degradation processes. A methodologically and mathematically sound set of tools is provided, which allows the treatment of the degradation of preventive barriers within a multistate modelling scheme for QRA. Application of the proposed approach to the slug catcher of a representative Upstream O\&G onshore plant has shown the benefits of using the multistate BNs methodology for the risk assessment of the unit. By benchmarking the results with those obtained by the BART methodology, it has been demonstrated that the BN allows to update the risk assessments in light of new KID becoming available, with the added value of the multivariate description of safety barriers HSs and FPs, describing the barrier performance evolution within a Living Risk Assessment framework.

A further extension of the work that is worth considering is the definition of an aggregated plant-level risk evaluation approach, by upscaling the here-proposed unit-level methodology (i.e., the slug catcher) to the plant level, in order to provide an aggregated risk profile for the whole plant where uncertainties and mutual dependencies 


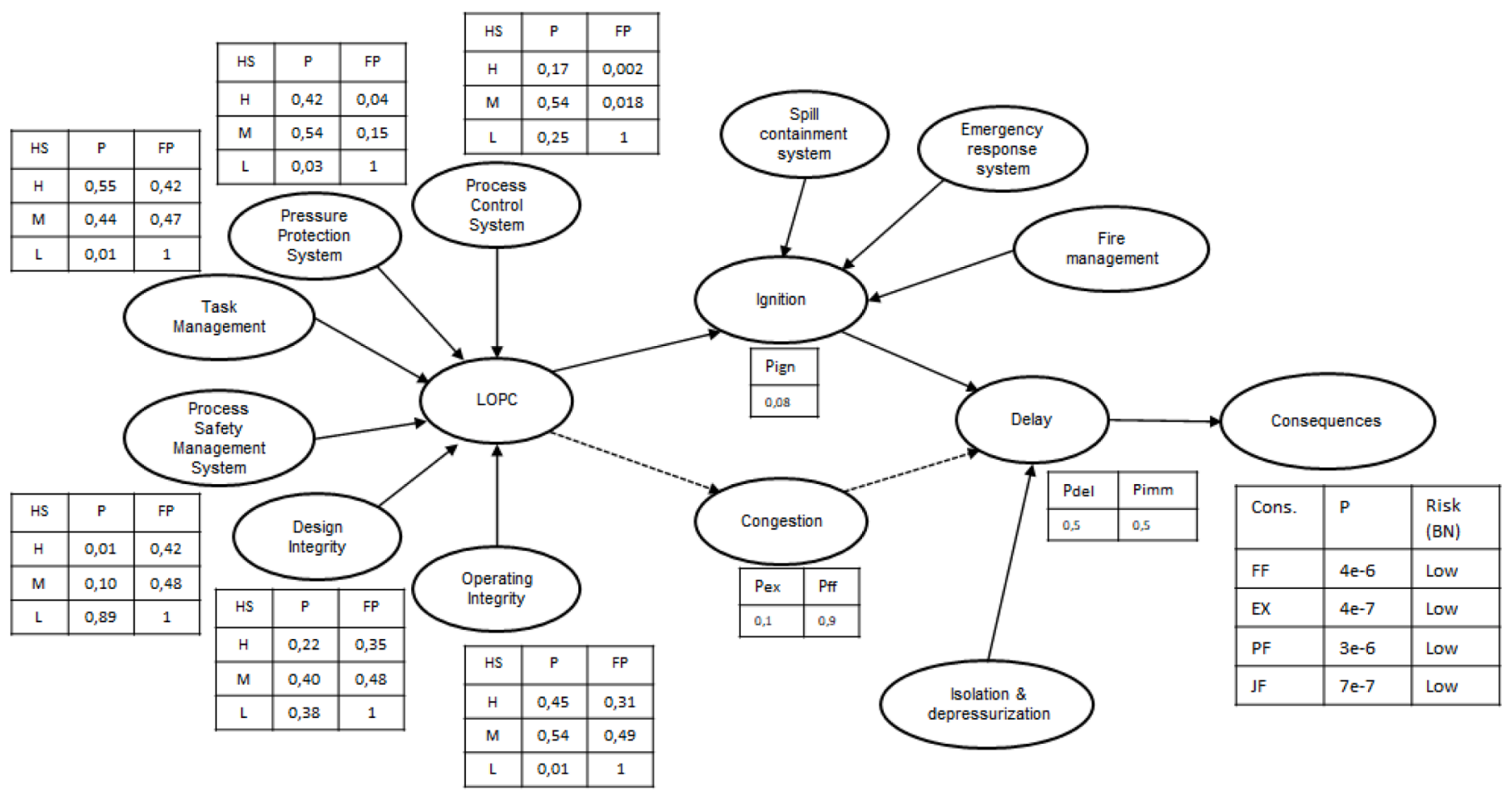

Figure 10. Updated BN of the slug catcher LOPC

Table 8

Values of the probabilities used in the BN

\begin{tabular}{lll}
\hline Probability & Value & Reference \\
\hline$P_{L O P C, 0}$ & 0.0241 & [22] \\
$P_{I g n}$ & 0.08 & (IP [24]) \\
$P_{D e l}$ & 0.5 & \\
$P_{I m m}$ & 0.5 & \\
$P_{E X}$ & 0.1 & \\
$P_{F F}$ & 0.9 & \\
$P_{P F}$ & $1 / 6$ & \\
$P_{J F}$ & $5 / 6$ & \\
\hline
\end{tabular}

\section{Authors statement}

All authors have equally contributed to the work.

\section{Declaration of Competing Interest}

None.

\section{Acknowledgments}

The authors gratefully acknowledge Eni HSEQ NR support, funding and direction given, under the multi-year BART-Bayesian Project.

among equipment and units are accounted for.

\section{APPENDIX A}

Given the baseline LOPC probability $P_{L O P C, 0}$, the actualized (reduced) probability of LOPC that accounts for the (positive) contribution of preventing is evaluated by total probability theorem, as in [39]. In practice, without loss of generality and for illustration purposes, let us consider the action of two preventive safety barriers (i.e., A and B), each one characterized by a set of HS probabilities, $\left\{P_{A}(H), P_{A}(M), P_{A}(L)\right\}$ and $\left\{P_{B}(H), P_{B}(M)\right.$, $\left.P_{B}(L)\right\}$ respectively, and the corresponding sets of FPs, $\left\{p_{A, H}, p_{A, M}, p_{A, L}\right\}$ and $\left\{p_{B, H}, p_{B, M}, p_{B, L}\right\}$. The actual LOPC probability $P_{L O P C}$ is then evaluated as:

$P_{L O P C}=\sum_{i \in\{H, M, L\} j \in\{H, M, L\}} P_{A}(i) \cdot p_{A, i} \cdot P_{B}(j) \cdot p_{B, j} \cdot P_{L O P C, 0}$

Finally, the probabilities of the consequences, FF, EX, PF and JF are evalutated as follows:

$$
\begin{aligned}
& P(F F)=P_{L O P C} \cdot P_{I g n} \cdot P_{D e l} \cdot P_{F F} \\
& P(E X)=P_{L O P C} \cdot P_{I g n} \cdot P_{D e l} \cdot P_{E X} \\
& P(P F)=P_{L O P C} \cdot P_{I g n} \cdot P_{I m m} \cdot P_{P F} \\
& P(J F)=P_{L O P C} \cdot P_{I g n} \cdot P_{I m m} \cdot P_{J F}
\end{aligned}
$$

where $P_{I m m}$ and $P_{D e l}$ are probability of immediate and delayed ignition, respectively, $P_{I g n}$ is the ignition probability following a LOPC, $P_{F F}$ the con- 
ditional probability of flash fire in case of delayed ignition, $P_{E X}$ the conditional probability of explosion in case of delayed ignition, $P_{P F}$ the conditional probability of pool fire in case of immediate ignition, and $P_{J F}$ the conditional probability of jet fire in case of delayed ignition (values are listed in Table 8).

\section{References}

[1] Abdolhamidzadeh B, Abbasi T, Rashtchian D, Abbasi SA. A new method for assessing domino effect in chemical process industry. J. Hazard. Mater. 2010;182. https://doi.org/10.1016/j.jhazmat.2010.06.049.

[2] Abimbola M, Khan F, Khakzad N, Butt S. Safety and risk analysis of managed pressure drilling operation using Bayesian network. Saf. Sci. 2015;76:133-44. https://doi.org/10.1016/j.ssci.2015.01.010.

[3] Adumene S, Khan F, Adedigba S, Zendehboudi S, Shiri H. Dynamic risk analysis of marine and offshore systems suffering microbial induced stochastic degradation. Reliability Engineering and System Safety 2021;207. March 2021 Article number 107388.

[4] Aneziris ON, Nivolianitou Z, Konstandinidou M. A Total Safety Management framework in case of a major hazards plant producing pesticides. Safety Science 2017;100:183-94. https://doi.org/10.1016/j.ssci.2017.03.021.

[5] Aneziris O, Papazoglou I. Bowties for occupational risk management. Total Safety and the Productivity Challenge. Routledge; 2019. p. 69-84. https://doi.org/ 10.4324/9781315108100-4.

[6] Bearfield G, Marsh W. Generalising Event Trees Using Bayesian Networks with a Case Study of Train Derailment. Computer Safety, Reliability, and Security 2005: 52-66. https://doi.org/10.1007/11563228 5.

[7] Bobbio A, Portinale L, Minichino M, Ciancamerla E. Improving the analysis of dependable systems by mapping fault trees into Bayesian networks. Reliability Engineering \& System Safety 2001;71(3):249-60. https://doi.org/10.1016/s0951 8320(00)00077-6.

[8] Cai B, Liu Y, Zhang Y, Fan Q, Liu Z, Tian X. A dynamic Bayesian networks modeling of human factors on offshore blowouts. Journal of Loss Prevention in the Process Industries 2013;26(4):639-49. https://doi.org/10.1016/j.jlp.2013.01.001.

[9] Cetinkaya, E.K. (2001). Reliability analysis of SCADA systems used in the offshore oil and gas industry.

[10] Cherubin P, Pellino S, Petrone A. Baseline risk assessment tool: A comprehensive risk management tool for process safety. Process Safety Progress 2011;30(3): 251-60. https://doi.org/10.1002/prs.10464.

[11] Cozzani V, Tugnoli A, Salzano E. The development of an inherent safety approach to the prevention of domino accidents. Accid. Anal. Prev. 2009;41. https://doi.org/ 10.1016/j.aap.2008.06.002.

[12] De Ruijter A, Guldenmund F. The bowtie method: A review. Safety Science 2016; 88:211-8. https://doi.org/10.1016/j.ssci.2016.03.001.

[13] Di Maio F, Rai A, Zio E. A dynamic probabilistic safety margin characterization approach in support of Integrated Deterministic and Probabilistic Safety Analysis. Reliability Engineering \& System Safety 2016;145:9-18. https://doi.org/10.1016/ j.ress.2015.08.016.

[14] Di Maio F, Scapinello O, Zio E, Ciarapica Alunni C, Decarli L, La Rosa L. A Multistate Bayesian Network for accounting the degradation of safety Barriers in the Living Risk Assessment of Oil and Gas Plants. In: Proceedings of the 30th European Safety and Reliability Conference and the 15th Probabilistic Safety Assessment and Management Conference, 21-26 June 2020; 2020. https://doi.org/ 10.3850/978-981-14-8593-0.

[15] Di Maio F, Scapinello O, Zio E, Ciarapica Alunni C, Decarli L, La Rosa L. A Novel KPI for Continuously Monitored Safety Barriers based on Probabilistic Safety Margins. In: Proceedings of the 30th European Safety and Reliability Conference and the 15th Probabilistic Safety Assessment and Management Conference, 21-26 June 2020; 2020. https://doi.org/10.3850/978-981-14-8593-0.

[16] Di Maio F, Scapinello O, Zio E, Ciarapica Alunni C, Decarli L, La Rosa L. Analytic Hierarchy Process for the Estimation of the Probability of Failures of Safety Barriers in Oil and Gas Installations. In: Proceedings of the 30th European Safety and Reliability Conference and the 15th Probabilistic Safety Assessment and Management Conference, 21-26 June 2020; 2020. https://doi.org/10.3850/978981-14-8593-0.

[17] Di Maio F, Fumagalli M, Guerini C, Perotti F, Zio E. Time-dependent reliability analysis of the reactor building of a nuclear power plant for accounting of its aging and degradation. Reliability Engineering \& System Safety 2021;205:107173. https://doi.org/10.1016/j.ress.2020.107173.

[18] Feng, W., Xiang, X., Jia, G., Dai, L., Gu, Y., Yang, X., ... Zhou, L. (2012). Applying the Quantitative Risk Assessment (QRA) to Improve Safety Management of Oil and Gas Pipeline Stations in China. Volume 4: Pipelining in Northern and Offshore Environments; Strain-Based Design; Risk and Reliability; Standards and Regulations. 10.1115/ipc2012-90130.

[19] Gertman D, Blackman H, Byers J, Haney L, Smith C, Marble J. (2005). The SPAR-H method. NUREG/CR-6883:US Nuclear Regulatory Commission.

[20] Goble R, Bier VM. Risk Assessment Can Be a Game-Changing Information Technology-But Too Often It Isn't. Risk Analysis 2013;33(11):1942-51. https:// doi.org/10.1111/risa.12055.

[21] Henley EJ, Kumamoto H. Probabalistic risk assessment: Reliability engineering, design, and analysis. New York: Institute of Electrical and Electronics Engineers; 1992.

[22] Health and Safety Executive. Offshore Hydrocarbon Releases Statistics. Health \& Safety Executive 2006. Available at: www.hse.gov.uk.
[23] Hollnagel E. Cognitive reliability and error analysis method: CREAM. New York: Elsevier; 1998.

[24] Research Report IP. 2006. www.energyinst.org.

[25] John A, Yang Z, Riahi R, Wang J. A risk assessment approach to improve the resilience of a seaport system using Bayesian networks. Ocean Eng 2016;111: 136-47. https://doi.org/10.1016/j.oceaneng.2015.10.048.

[26] Kalantarnia M, Khan F, Hawboldt K. Dynamic risk assessment using failure assessment and Bayesian theory. Journal of Loss Prevention in the Process Industries 2009;22(5):600-6. https://doi.org/10.1016/j.jlp.2009.04.006.

[27] Khakzad N, Khan F, Amyotte P. Dynamic safety analysis of process systems by mapping bow-tie into Bayesian network. Process Safety and Environmental Protection 2013;91(1-2):46-53. https://doi.org/10.1016/j.psep.2012.01.005.

[28] Khakzad N, Reniers G, Abbassi R, Khan F. Vulnerability analysis of process plants subject to domino effects. Reliability Engineering \& System Safety 2016;154: 127-36. https://doi.org/10.1016/j.ress.2016.06.004.

[29] Khakzad N. Which Fire to Extinguish First? A Risk-Informed Approach to Emergency Response in Oil Terminals. Risk Analysis 2018;38(7):1444-54. https:// doi.org/10.1111/risa.12946.

[30] Khakzad N, Landucci G, Cozzani V, Reniers G, Pasman H. Cost-effective fire protection of chemical plants against domino effects. Reliability Engineering and System SafetyOpen Access 2021;169:412-21. January 2018.

[31] Khan FI, Sadiq R, Husain T. Risk based process safety assessment and control measures design for offshore process facilities. Journal of Hazardous Materials 2002;A94:1-36. https://doi.org/10.1016/s0304-3894(02)00004-3.

[32] Kirwan B. The validation of three human reliability quantification techniques THERP, HEART and JHEDI: Part 1 - technique descriptions and validation issues. Applied Ergonomics 1996;27(6):359-73. https://doi.org/10.1016/s0003-6870 (96)00045-2.

[33] Li X, Chen G, Khan F, Xu C. Dynamic risk assessment of subsea pipelines leak using precursor data. Ocean Engineering 2019;178:156-69.

[34] Li X, Zhang Y, Abbassi R, Yang M, Zhang R, Chen G. Dynamic probability assessment of urban natural gas pipeline accidents considering integrated external activities. Journal of Loss Prevention in the Process Industries 2021;69:104388.

[35] Mancuso A, Compare M, Salo A, Zio E. Portfolio optimization of safety measures for reducing risks in nuclear systems. Reliability Engineering \& System Safety 2017; 167:20-9. https://doi.org/10.1016/j.ress.2017.05.005.

[36] Mkrtchyan L, Podofillini L, Dang VN. Bayesian belief networks for human reliability analysis: A review of applications and gaps. Reliability Engineering \& System Safety 2015;139:1-16. https://doi.org/10.1016/j.ress.2015.02.006.

[37] Nutt WT, Wallis GB. Evaluation of nuclear safety from the outputs of computer codes in the presence of uncertainties. Reliability Engineering \& System Safety 2004;83(1):57-77. https://doi.org/10.1016/j.ress.2003.08.008.

[38] OREDA. Offshore Reliability Data Handbook. Trondheim, Norway: DNV; 2002.

[39] Pearl J. Fusion, propagation, and structuring in belief networks. Artificial Intelligence 1986;29(3):241-88. https://doi.org/10.1016/0004-3702(86)90072-x.

[40] Petrone A, Scataglini L, Cherubin P. B.A.R.T (BAseline Risk Assessment Tool): A Step Change in Traditional Risk Assessment Techniques for Process Safety and Asset Integrity Management. In: SPE Annual Technical Conference and Exhibition; 2011. https://doi.org/10.2118/146845-MS.

[41] Puga-Leal R, Lopes Pereira Z. Process capability in services. International Journal of Quality \& Reliability Management 2007;24(8):800-12.

[42] Ramzali N, Lavasani MRM, Ghodousi J. Safety barriers analysis of offshore drilling system by employing Fuzzy Event Tree Analysis. Safety Science 2015;78:49-59. https://doi.org/10.1016/j.ssci.2015.04.004.

[43] Villa V, Paltrinieri N, Khan F, Cozzani V. Towards dynamic risk analysis: A review of the risk assessment approach and its limitations in the chemical process industry. Safety Science 2016;89:77-93. https://doi.org/10.1016/j. ssci.2016.06.002.

[44] Xing J, Zeng Z, Zio E. A framework for dynamic risk assessment with condition monitoring data and inspection data. Reliability Engineering \& System Safety 2019:106552. https://doi.org/10.1016/j.ress.2019.106552.

[45] Zeng T, Chen G, Yang Y, Chen P, Reniers G. Developing an advanced dynamic risk analysis method for fire-related domino effects. Process Safety and Environmental Protection 2019. https://doi.org/10.1016/j.psep.2019.11.029.

[46] Zeng Z, Zio E. Dynamic Risk Assessment Based on Statistical Failure Data and Condition-Monitoring Degradation Data. IEEE Transactions on Reliability 2018;67 (2):609-22. https://doi.org/10.1109/tr.2017.2778804.

[47] Zio Enrico. On the use of the analytic hierarchy process in the aggregation of expert judgments. Reliability Engineering \& System Safety 1996;53:127-38. https://doi. org/10.1016/0951-8320(96)00060-9.

[48] Zio E, Cantarella M, Cammi A. The analytic hierarchy process as a systematic approach to the identification of important parameters for the reliability assessment of passive systems. Nuclear Engineering and Design 2003;226(3): 311-36. https://doi.org/10.1016/s0029-5493(03)00211-5. 
[49] Zio E, Podofillini L, Baraldi P, Dang V, Librizzi M, Conti M. A Bayesian network model for dependence assessment in human reliability analysis. Reliability, Risk, and Safety 2009. https://doi.org/10.1201/9780203859759.ch32.

[50] Zio E, Di Maio F, Tong J. Safety margins confidence estimation for a passive residual heat removal system. Reliability Engineering \& System Safety 2010;95(8) 828-36. https://doi.org/10.1016/j.ress.2010.03.006.
[51] Zio Enrico. Some Challenges and Opportunities in Reliability Engineering. IEEE Transactions on Reliability 2016;65(4):1769-82. https://doi.org/10.1109/ tr.2016.2591504. 